\title{
Genetic studies of laboratory reared Mytilus edulis. I. Genotype specific selection in relation to salinity
}
A. R. Beaumont,
C. M. Beveridge,
E. A. Barnet,
M. D. Budd and
M. Smyth-Chamosa

School of Ocean Sciences, UCNW, Marine Science Laboratories, Menai Bridge, Gwynedd LL59 5EY.

\begin{abstract}
Significant deviations from the Hardy-Weinberg equilibrium in natural populations of bivalves may be due to a number of factors such as the Wahlund effect, null alleles and selection, and it is often difficult to decide which of these factors is operating. The use of laboratory controlled single crosses and mass matings, where the genotypes of the parents are known, can provide strong evidence for selection when deviations from expected genotype frequencies are observed in the offspring. Several cultures of offspring from a single cross and from a mass mating of the mussel, Mytilus edulis, were reared through the larval stage at either 32 or 25 parts per thousand salinity. At the early post-larval stage salinities were altered for some cultures while others remained unchanged. Samples were taken for electrophoresis as post-larvae and again as juveniles and were scored at the Lap, Pgm, Odh, Hex, Pgk and Gpi loci. Significant deviations from expected frequencies were observed at many loci in the offspring but these deviations did not appear to be related to salinity except at the $L a p$ locus. The allele $L_{a p}{ }^{94}$ was selected against at low salinity during the post-larval to juvenile stage, but no such selection occurred at low salinity during the larval stage. The data fit Koehn's (1985) model of post-larval selection at the Lap locus in mussels in Long Island Sound, USA, but suggest that there may be ontogenetic variation in fitness at this locus. Results at the Pgm and Gpi loci indicate that these loci are probably acting as markers for other loci on the same chromosome which have a strong effect on fitness. Density dependent factors may have caused some of the non-random mortality amongst offspring which was apparently independent of salinity.
\end{abstract}

\section{INTRODUCTION}

Considerable data now exist concerning the population genetics of mussels derived from studies on taxonomy (Skibinski et al., 1983), selection (Levinton and Fundiller, 1975; Koehn, 1983; Koehn, 1985), migration (Koehn et al., 1976) population structure (Tracey et al., 1975) heterozygosity and growth rate (Koehn and Gaffney, 1984), heterozygosity and oxygen consumption (Diehl et al., 1985) and heterozygote deficiencies (Zouros and Foltz, 1984). However, studies on natural populations suffer from the fact that observed deviations from the Hardy-Weinberg equilibrium at enzyme loci may be caused by a variety of forces, notably, meiotic drive, inbreeding, null alleles, the mixing of larval populations (Wahlund effect) or selection. Consequently it is often difficult to specify one or other of these forces as the causative agent in deviations from the Hardy Weinberg equilibrium.
Some of the problems associated with interpreting data from natural populations can be overcome by the use of single families or controlled mass matings with the subsequent experimental growth of cohorts of larvae and spat in the laboratory. This enables precise prediction of phenotype proportions amongst offspring and any significant deviations from predicted proportions are likely to be due to phenotype-specific mortality. Studies using laboratory reared single families and mass matings of mussels and other bivalves have already provided evidence of direct or indirect selection at a number of enzyme loci (Beaumont et al., 1983; Adamkewicz et al., 1984; Hvilsom and Theisen, 1984; Mallet et al., 1985; Gaffney and Scott, 1984; Mallet et al., 1986).

In a series of experiments using single families and a mass mating of Mytilus edulis we have investigated the effects of salinity and temperature on genotype-specific mortalities at both the late 
spat and the juvenile stages of development. This paper details the genetic consequences of the trials involving salinity variations applied during the rearing of larvae and juveniles.

Koehn and colleagues (reviewed in Koehn, 1985) have documented a thorough investigation into the role of salinity in selection at the leucine amino-peptidase locus (Lap) in $M$. edulis from Long Island Sound, USA. Their findings indicate that individual mussels exhibiting the allele $\operatorname{Lap}^{94}$ are poorly adapted to cope with the physiological stresses encountered at reduced salinity and are therefore selected against in areas of low salinity in Long Island Sound.

Some of the cultures reared in our experiments approximately mimic the changing salinity experienced by $M$. edulis larvae and spat in Long Island Sound, and the results described here provide some evidence to support Hilbish and Koehn's (1985a) conclusions on selection at the Lap locus in reduced salinity.

\section{MATERIALS AND METHODS}

Ripe $M$. edulis were collected from the underside of permanently moored barges in the Menai Strait, North Wales, U.K. during spring 1984 and 1985 and were held in flowing sea water at a temperature of $7 \pm 1^{\circ} \mathrm{C}$ until required for spawning. In the first experiment (M1) a mating was performed between one male and one female while in the second experiment (MM) a mass mating was performed using nine males and six females. Mussels were induced to spawn by injecting $0.5 \mathrm{M} \mathrm{KCl}$ into the mantle cavity and leaving the mussels emersed for 30 minutes. In the case of the mass mating, considerable care was taken to ensure numerically equal gametic contributions from each individual to the gamete pool at fertilisation. As and when each mussel had spawned sufficient gametes, counts were made of egg numbers, using a Coulter Counter, and of spermatozoa using a haemacytometer. When a suitable number of mussels had spawned, spermatozoa were mixed into a suspension which contained equal proportions of gametes from each male. Similarly an egg suspension was prepared containing equal proportions of eggs from each female. The two suspensions were then mixed to allow random fertilisation of the gametes.

Cultures of larvae were reared through the veliger stage in 5-litre pyrex glass jars using the method of Bayne (1965). Larvae were held at a concentration of approximately $5 \mathrm{ml}^{-1}$ in $0.2 \mu \mathrm{m}$ filtered and UV treated sea-water. The sea-water was changed three times each week and larvae were fed a mixture of Pavlova lutheri (Droop) Green at 40 cells $\mu \mathrm{l}^{-1}$ and Rhodomonas baltica Karsten at 10 cells $\mu l^{-1}$. Water of low salinity was prepared by dilution of filtered sea-water with distilled water and salinity was checked using a portable refractometer.

When 50 per cent of the larvae in a culture had reached metamorphosis the larvae and post-larvae in that culture were retained in a cylindrical plastic container, closed at the base with $170 \mu \mathrm{m}$ mesh, which fitted loosely into the jar. Using this system, both metamorphosed and unmetamorphosed larvae were held in identical conditions and larvae which were slow to metamorphose were not lost from the culture. This avoids the problem of artificial selection for early metamorphosing larvae in laboratory cultures. Mussels were retained in this system for the duration of the experiment with a weekly water change and with food addition three times a week. The ration of food was doubled from 50 cells $\mu \mathrm{l}^{-1}$ to 100 cells $\mu \mathrm{l}^{-1}$ after the postlarvae were sampled for electrophoresis and occasionally one of the algal species was fed exclusively when the other species was not available.

The experimental procedure for varying salinity was as follows. In the single family (M1) the larvae were divided at the " $D$ " larva stage (three days after fertilisation) into four separate cultures. Two of these cultures were reared at 25 parts per thousand salinity (M1C, M1D) and the other two at 32 parts per thousand salinity (M1A, M1B). After metamorphosis, postlarvae were grown for a short period until they became large enough for samples to be genotyped using micro-electrophoresis $(500-1000 \mu \mathrm{m})$. After this first sampling one of the low salinity cultures was changed to high salinity (M1C) and one of the high salinity cultures (M1B) was changed to low salinity. All four cultures were then grown on to the juvenile stage when further samples were taken for genetic analysis. A similar procedure was adopted with four cultures derived from the mass mating (MMA, MMB, MMC and MMD).

In the case of the culture MMD, which was reared exclusively at low salinity, electrophoretic analysis was only carried out at the juvenile stage. This culture was then subjected to a regime which consisted of removing the mussels from water for a 2 hour period each weekday for a period of one month. After this time a further sample was taken for electrophoresis (MMD, postemersion). 


\section{ELECTROPHORESIS}

Micro-electrophoresis of post-larvae was carried out using cellulose acetate gels in a Shandon electrophoresis chamber. Samples were prepared by placing individual mussels in $5 \mu \mathrm{l}$ of 20 per cent glycerol in $0.1 \mathrm{M}$ Tris/HCL buffer on the sampling pads of a Shandon applicator. The samples were then macerated with a fine glass rod and immediately transferred, eight at a time, onto the gel by repeated application of a Shandon multisample applicator. Electrophoresis was carried out using a $22 \mathrm{mM}$ tris- $135 \mathrm{mM}$ glycine buffer, $p \mathrm{H} 8 \cdot 6$, for a run time of from 30 to 45 minutes at 200 volts. Starch gel electrophoresis was carried out on whole, crushed juvenile mussels, using the scheme described in Beaumont and Beveridge (1983) and Beaumont et al. (1983).

The enzymes investigated were leucine amino peptidase (LAP, EC 3.4.11.-), phosphoglucomutase (PGM, EC 2.7.5.1.), octopine dehydrogenase $(\mathrm{ODH}, \mathrm{EC}$ 1.5.1.11.), $\beta$-n-acetylglucoseaminidase (HEX, EC 3.2.1.30), phosphoglycerate kinase (PGK, EC 2.7.2.3.), diaphorase (cytochrome $b$ reductase) (DIA, EC 1.6.2.2.) and glucose phosphate isomerase (GPI, EC 5.3.1.9.). Staining recipes for most enzymes were taken from Harris and Hopkinson (1976) except that 0.8 per cent Meldola blue was used instead of PMS (Turner and Hopkinson, 1979); ODH and LAP were stained using the recipes described by Beaumont et al. (1980) and Beaumont et al. (1983) respectively. Stains were overlayed in agar or filter paper on the starch gels but liquid stains were used for the cellulose acetate gels.

Increased variation can be detected at the Pgm locus in mussels by using a tris-maleic buffer at $p \mathrm{H} 6.0$ rather than $p \mathrm{H} \mathrm{7.4}$ (Beaumont \& Beveridge, 1983). This increased variation could not be detected on cellulose acetate gels using the tris-glycine buffer, even if the $p \mathrm{H}$ was reduced, so some samples of larger post-larvae from the family M1 were genotyped on thin layer $(2 \mathrm{~mm})$ starch gels with tris-maleic buffer at $p \mathrm{H} \mathrm{6.0.} \mathrm{Spat} \mathrm{from} \mathrm{the}$ mass mating cultures were not analysed at the Pgm locus.

Due to the small size of post-larvae, each individual could only be analysed at 2 enzyme loci on cellulose acetate gels, and it was also necessary to increase the concentrations of chemicals in the staining cocktails.

At most loci alleles are notated by a superscript which describes their mobility as a percentage of the mobility of the commonest allele in local wild populations of $M$. edulis. Notation of the alleles at the Lap locus follows that of Koehn et al. (1976). Specimens from family M1 have been run on the same gel as $M$. edulis from Stony Brook and our designation of Lap alleles in relation to Koehn et al.'s (1976) data has been confirmed (McDonald, personal communication). Gpi allele notation follows that given by Ahmad et al. (1977).

Expected allele and genotype frequencies in the family M1 were calculated from the known genotypes of the parents. In the case of the mass mating an $18 \times 12$ table was drawn up for each locus to calculate all the possible genotypes and their frequencies assuming random mixing of all the gametes from the 15 progenitor mussels. The significance of deviations of observed from expected genotype and allele frequencies at each locus for individual samples was calculated using the $\chi^{2}$ goodness of fit test. For the purposes of $\chi^{2}$ analysis, genotype classes were pooled, where necessary, to ensure that only a small proportion of expected values were less than 5 and that none were less than unity. For each locus, heterogeneity of genotype frequencies over all samples of spat and juveniles was tested using the $\chi^{2}$ contingency test.

\section{RESULTS}

Genotype frequency and allele frequency data at the Lap, Odh, Pgm, Gpi, Pgk and Dia loci for the single family (M1) and the mass mating (MM) are presented in tables 1-3.

\section{Leucine aminopeptidase (Lap)}

There were no significant deviations from expected allele or genotype frequencies at either the spat or juvenile stage in the single family culture reared at high salinity (M1A, table 1 ). By contrast, in the equivalent mass mating culture (MMA) the genotypes $\operatorname{Lap}^{94 / 96}$ and $L a p^{94 / 98}$ were in excess at the spat stage with $L a p^{98 / 98}$ occurring at a significantly higher frequency than expected at the juvenile stage (table 2). The allele $\operatorname{Lap}^{94}$ was in excess at the spat stage with both $\operatorname{Lap}^{94}$ and $L a p^{98}$ in excess at the juvenile stage (table 2).

In the single family culture reared to spat at high salinity and then transferred to low salinity (M1B) no significant deviations were observed from expected allele or genotype frequencies (table 1). In the equivalent mass mating culture (MMB) a significant excess of the $L a p^{94 / 96}$ genotype was observed at the spat stage but the sample taken at 


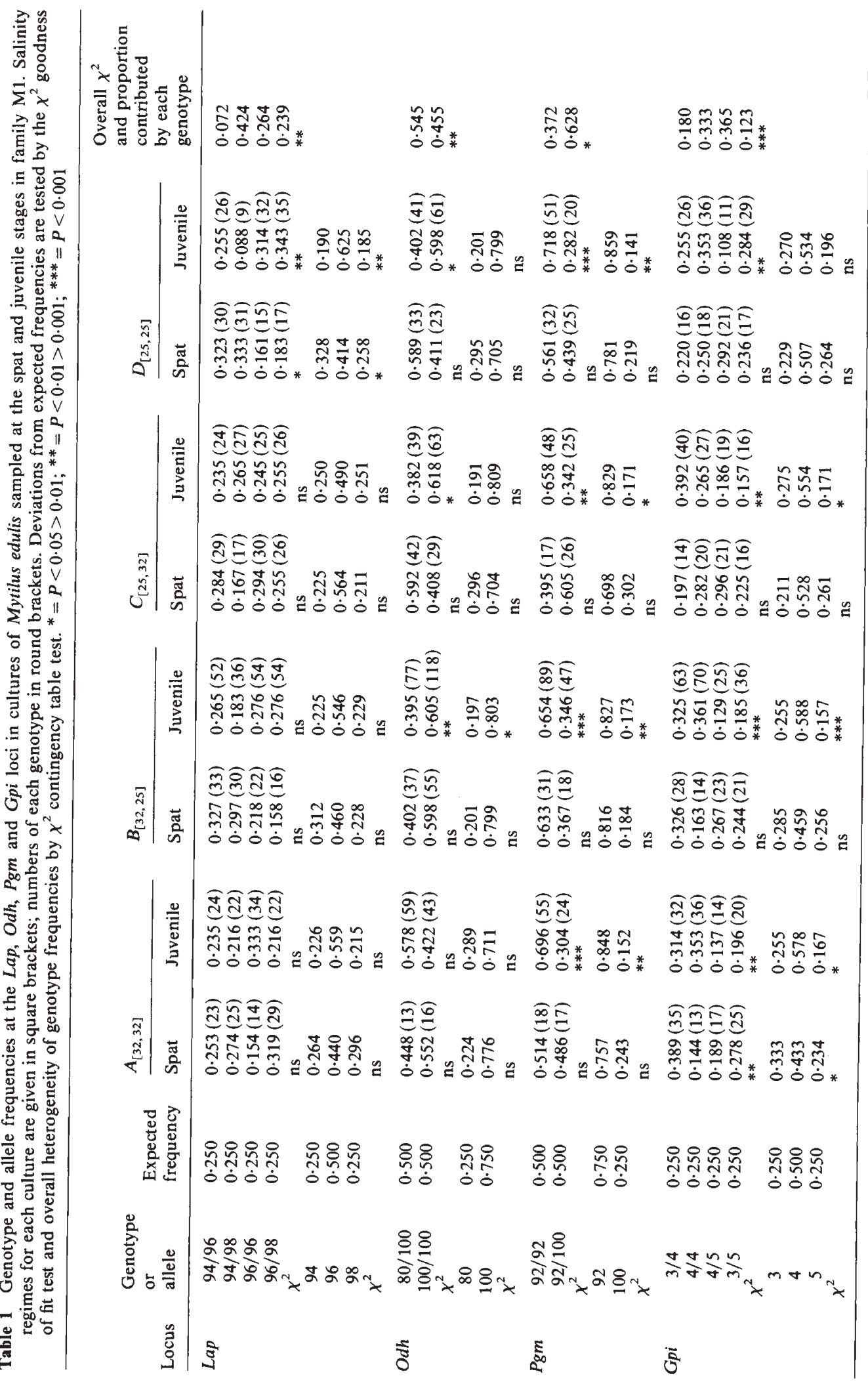




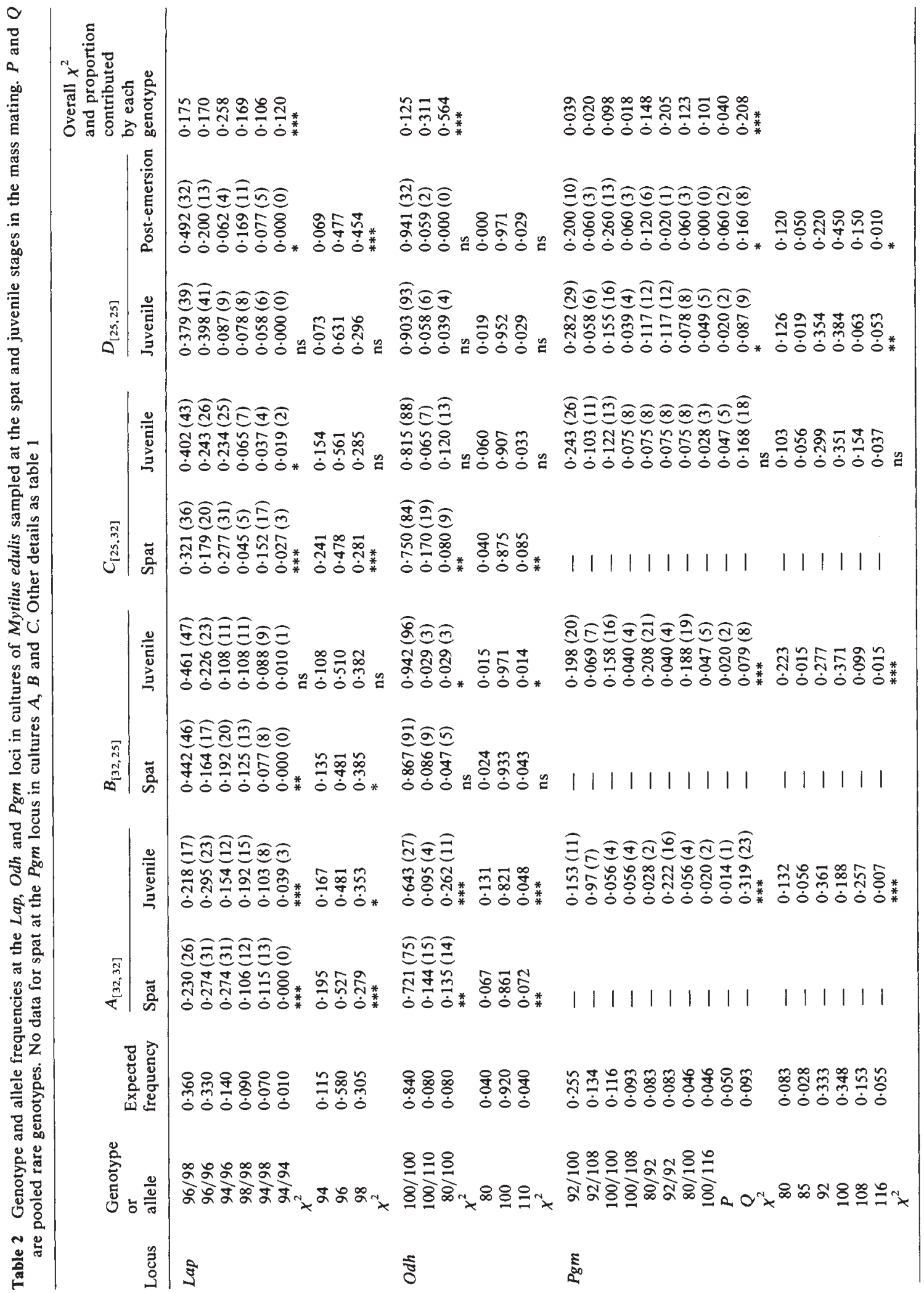


the juvenile stage revealed no significant deviations from expected genotype frequencies (table 2). Allele frequency data indicate that the Lap $^{94}$ excess observed at the spat stage was no longer found in the juveniles.

Samples from the single family culture reared to spat at low salinity and then transferred to high salinity (M1C) revealed no significant deviations in Lap geneotype or allele frequencies (table 1). In the equivalent culture (MMC, table 2) the genotypes $L a p^{94 / 96}$ and $L a p^{94 / 98}$ were significantly in excess at the spat stage. This resulted in a significant excess of the allele $L a p^{94}$. At the juvenile stage the $L_{a p}{ }^{94}$ allele still occurred at a higher frequency than expected, but not significantly so.

The only culture from the single family which showed significant differences from expected Lap genotype or allele frequencies was the culture reared entirely at low salinity (M1D, table 1). In this culture the genotypes $L a p^{94 / 96}$ and $L a p^{94 / 98}$ were significantly in excess at the spat stage resulting in a significant excess of the $\operatorname{Lap}^{94}$ allele. By contrast, the sample taken from the juveniles revealed a significant deficit of the $\operatorname{Lap}^{94 / 98}$ genotype and a significant deviation in allele frequencies due to a deficit of $L a p^{94}$ and an excess of $L a p^{96}$. In the culture (MMD, table 2), no significant deviations from expected Lap genotype or allele frequencies were observed at the juvenile stage. However, the periods of aerial exposure produced a significant reduction in the genotype $L a p^{94 / 96}$, and resulted in a significant deficiency of the $L a p^{94}$ and an excess of the $\operatorname{Lap}^{98}$ alleles.

Significant overall heterogeneity of Lap genotype frequencies was apparent in both the single family and the mass mating $\left(\mathrm{M} 1, \chi_{21}^{2}=\right.$ 46.552, $P<0.01>0.001 ; \mathrm{MM}, \chi_{35}^{2}=101 \cdot 185, P<$ $0 \cdot 001$ ) however this heterogenity was more evident at the spat stage than at the juvenile stage in the single family (spat stage alone, $\chi_{9}^{2}=19 \cdot 588, P<$ $0.05>0.01$; juvenile stage alone, $\chi_{9}^{2}=14 \cdot 34$, ns). Significant heterogenity was present at both the spat and juvenile stages of the mass mating (spat, $\chi_{10}^{2}=29.79, P<0.001$; juvenile $\chi_{20}^{2}=49 \cdot 47, P<$ $0 \cdot 001)$.

\section{Octopine dehydrogenase (Odh)}

In the single family culture M1A (table 1), no significant deviations from expected genotype or allele frequencies were observed. However, in MMA (table 2), a significant deficiency of the allele $O d h^{100}$ was evident at both the spat and juvenile stages. No significant deviations were observed at the spat stage in the single family culture M1B or the mass mating culture MMB. When these cultures were sampled after post-spat growth at low salinity, however, the allele $O d h^{100}$ was found to be significantly in excess (tables 1 and 2). The single family culture, M1C, revealed no significant deviation from expected genotype or allele frequencies as spat but when sampled as juveniles, after a period of growth at high salinity an excess of the genotype $O d h^{100 / 100}$ was observed (table 1). By contrast, MMC (table 2) showed a significant $O d h^{100}$ deficit in the spat, which was no longer evident in juveniles. Cultures reared entirely at low salinity (M1D and MMD, tables 1 and 2) were generally in good agreement with expectations although the $O d h^{100 / 100}$ genotype was more frequent than expected after the spat stage. There was significant overall heterogeneity of $O d h$ genotype frequencies in the single family and mass mating cultures (M1 $\chi_{7}^{2}=22 \cdot 853, P<0 \cdot 01>0.001$; MM $\left.\chi_{14}^{2}=56.089 P<0 \cdot 001\right)$. Approximately 56 per cent of the $\chi^{2}$ value in the mass mating was derived from the $O d h^{80 / 100}$ genotype class and 31 per cent from the $O d h^{100 / 100}$ class. Both these genotypes were expected at low frequencies but in the contingency table only 17 per cent of the expected values were less than five and none less than $2 \cdot 8$, and we are therefore satisfied that the $\chi^{2}$ result is valid. In the single family significant heterogeneity was found at both the spat and the juvenile stages (spat $\chi_{3}^{2}=7.98, P<0.50>0.01$; juvenile $\left.\chi_{3}^{2}=11.41, P<0.01>0.001\right)$ whereas significant heterogeneity was not evident until the juvenile stage in the mass mating (spat $\chi_{4}^{2}=9 \cdot 20$, ns; juvenile $\left.\chi_{8}^{2}=34 \cdot 22, P<0 \cdot 001\right)$.

\section{Phosphoglucomutase (Pgm)}

Due to the problems of resolution of all alleles at the Pgm locus (Beaumont and Beveridge, 1983) no Pgm data were collected from the spat of the mass mating cultures. In no cultures of the single family were significant differences from expected genotype or allele frequencies observed at the spat stage, but in every culture, irrespective of salinity treatments the homozygote class $\mathrm{Pgm}^{92 / 92}$ was significantly in excess at the juvenile stage (table 1). In the mass mating a total of 17 different genotypes were observed. Although the $P g m^{92 / 92}$ homozygote was significantly in excess at the juvenile stage in culture MMA (table 2) the allele showing the greatest excess was $\mathrm{Pgm}^{108}$. By contrast in culture MMB there was a significant excess of allele $P g m^{80}$ and a deficiency of $\mathrm{Pgm}^{92}$ at the juvenile stage. An excess of $\mathrm{Pgm}^{80}$ was also noted both at the juvenile stage and after aerial exposure in the culture MMD 
which was reared entirely at low salinity. Overall heterogeneity of $\mathrm{Pgm}$ genotype frequencies was significant in the single family and mass mating cultures $\quad\left(\mathrm{M} 1 \quad \chi^{2}=17.716, \quad P<0.05>0.01\right.$; $\left.\mathrm{MM} \chi_{36}^{2}=95.627, \quad P<0.001\right)$. No single low frequency genotype class contributed a large fraction of the total mass mating $\chi^{2}$ value. When the spat and juvenile stages were tested separately for heterogeneity in the single family, neither stage was significant on its own (spat $\chi_{3}^{2}=5 \cdot 46$, ns; juvenile $\chi_{3}^{2}=1 \cdot 13$, ns). This merely confirms that the same temporal trend from spat frequencies to juvenile frequencies was shared by all cultures.

\section{Glucosephosphate isomerase (Gpi)}

In culture M1A, there was a significant deficiency of the allele $G p i^{4}$ resulting from a reduction against expected numbers of the genotypes $\mathrm{Gpi}^{4 / 4}$ and $\mathrm{Gpi}^{4 / 5}$ (table 1). The situation was different in samples taken from the juveniles where there was an excess of allele $G p i^{4}$ and a deficiency of allele $G p i^{5}$. Culture MMA showed significant variation in allele frequencies at the spat stage with $G p i^{4}$ being less frequent than expected, but no significant allele frequency differences were present at the juvenile stage (table 3 ).

In cultures MIB, MIC and MID, there was little variation from expected genotype and allele frequencies at the spat stage but the allele $G p i^{5}$ was generally deficient when cultures were sampled as juveniles (table 1). By contrast, data from culture MMB revealed a significant excess of $G p i^{5}$ in juveniles. Culture MMC showed a deficit of $G p i^{5}$ at the spat stage but an excess of $G p i^{6}$ and a deficiency of $G p i^{1}$ at the juvenile stage (table 3 ). There was significant overall Gpi genotype frequency heterogeneity within the single family and the mass mating (M1 $\chi_{21}^{2}=55 \cdot 309, P<0 \cdot 001$; MM $\left.\chi_{42}^{2}=122.395, \quad P<0.001\right)$. There was, however, no significant between culture variation at the spat, or juvenile stages in the single family (spat $\chi_{9}^{2}=14 \cdot 68$, ns; juvenile $\chi_{9}^{2}=12 \cdot 31$, ns) and this suggests, as with the Pgm locus, that genotype frequency trends from spat to juvenile were similar in most cultures. In the mass mating, however, significant variation was evident at both stages (spat $\chi_{12}^{2}=26 \cdot 48, P<0 \cdot 001$; juvenile $\chi_{24}^{2}=88 \cdot 37$, $P<0.001$ ).

\section{Phosphoglycerate kinase (PgK)}

No variation was present at the $P g k$ locus in the single family. In culture MMA, there was a significant deficiency of the allele $P g k^{100}$ at the spat stage due to fewer than expected $P g k^{100 / 100}$ and $P g k^{100 / 115}$ individuals being present in the sample (table 3). The juveniles, however, showed no significant deviation from expected allele or genotype frequencies. The culture MMB showed a significant excess of $P g k^{100}$ at both the spat and the juvenile stages. Cultures $\mathrm{MMC}$ and $\mathrm{D}$ revealed minor or insignificant deviations from expected allele and genotype frequencies. However, the contingency table $\chi^{2}$ test showed significant overall heterogeneity in genotype frequencies $\left(\chi_{21}^{2}=\right.$ 76.796, $P<0.001)$ although between culture variations at the spat and juvenile stages, when analysed separately were not significant (spat $\chi_{6}^{2}=$ $19 \cdot 39$, ns; juvenile $\chi_{12}^{2}=18 \cdot 29$, ns),

\section{Diaphorase (Dia)}

No variation was present at the Dia locus in the single family. Little variation was evident in the two cultures of the mass mating which were reared at constant salinity (MMA and D, table 3). However, in culture MMB a significant deficiency of the common homozygote Dia ${ }^{100 / 100}$ at both the spat and juvenile stages resulted in a significant overall deficiency of the allele $\mathrm{Dia}^{100}$. A deficiency of $\mathrm{Dia}^{100}$ was also evident in the culture MMC at the juvenile stage. Significant heterogeneity in genotype frequencies were found at the spat stage, the juvenile stage and also over all cultures (spat $\chi_{4}^{2}=23.58, \quad P<0.001 ;$ juvenile $\chi_{8}^{2}=21.95, \quad P<$ $0.01>0.001$; overall $\left.\chi_{28}^{2}=86 \cdot 26, P<0.001\right)$.

\section{DISCUSSION}

Before discussing the results it is necessary to establish whether each mussel involved in the mass mating contributed equally to the production of zygotes since the calculation of expected genotype frequencies is based on equal contribution of all progenitors. Gaffney and Scott (1984) conducted genetic analyses of mass matings in a number of bivalves and concluded that unequal contributions of the progenitors was a common feature of all their mass matings. They do not, however, give full details of the methodology employed to ensure that equal numbers of gametes from each progenitor were mixed. In our experiment gametes from all females were mixed evenly, then equal numbers of gametes from all males were mixed and finally the suspension of spermatzoa was added to the suspension of eggs. As far as possible, therefore, each gamete had an equal chance of coming into contact with every other gamete. 


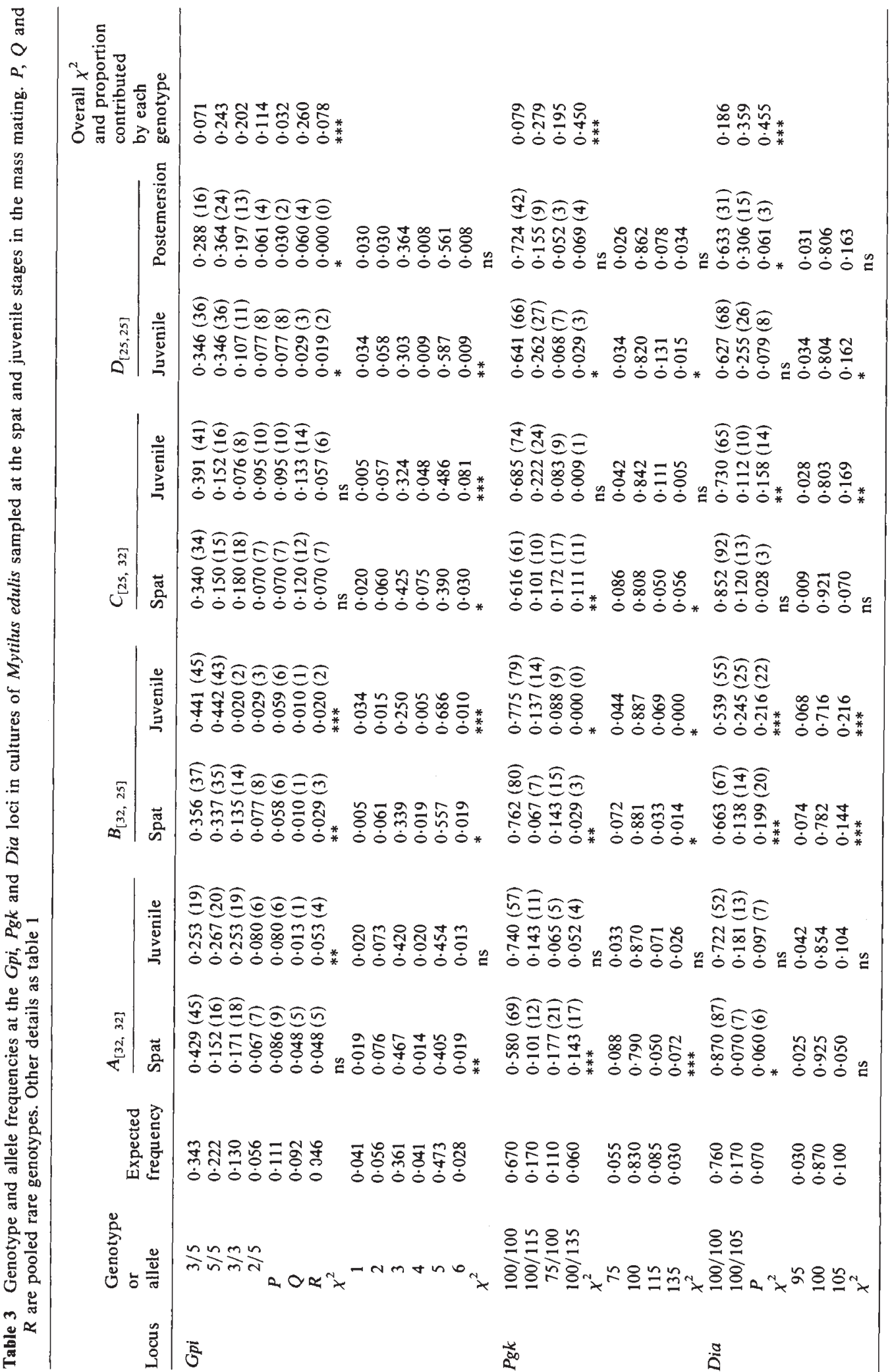


If equal contributions were made by each progenitor, and in the absence of selection, agreement with predicted ratios of genotype frequencies would be expected at all loci. Of the total of 59 loci examined in 8 cultures of the mass mating (four cultures of which were subjected to different temperature regimes and are not discussed in this paper), in 27 cases genotype frequencies did not differ significantly from the calculated frequencies. These 27 non-significant results are distributed fairly evenly amongst loci (Lap, 3; Odh, 6; Pgm, 1; Gpi, 5; Pgk, 7; Dia, 5) which suggests that equal contributions were made by all progenitors. Furthermore, all predicted genotypes, even very rare ones at the Gpi and Pgm loci, were observed at least once.

Unequal contribution of progentiors would lead to consistent deficiencies of particular genotypes at each locus. Examination of the data (Tables 2 and 3 ) reveals that at no loci are specific genotypes consistently deficient, and the considerable heterogeneity of genotype frequencies at the spat stage further support this conclusion.

As a further test of equal contributions of gametes in the mass mating mean frequencies of alleles at the five loci tested at the spat stage were calculated from "control" cultures which had been reared at high salinity and $15^{\circ} \mathrm{C}$. No single progenitor mussel introduced alleles at all loci which were either consistently deficient or in excess amongst the offspring. Because the mussels used in the mass mating did not all spawn at the same time, gametes from some individuals were, of necessity, held longer than others before fertilisation. According to Sprung and Bayne (1984) mussel eggs remain fertilisable for up to 6 hours at $19^{\circ} \mathrm{C}$ and complete loss of mobility of sperms only occurs after 11 hours at that temperature. In our experiment, the maximum delay of fertilisation was $3 \frac{1}{4}$ hours for eggs and $4 \frac{3}{4}$ hours for sperm. It is therefore unlikely that aging of gametes from the first mussels to spawn would have caused unequal contribution of those gametes in the zygotes. Furthermore alleles present in the earliest mussels to spawn were not consistently deficient amongst the offspring. Therefore, although it is not possible to state with absolute certainty that equal contributions were made by each progenitor in the mass mating, all the evidence points to equal rather than unequal contributions.

According to Koehn (1985) and Hilbish and Koehn $(1985 a, b)$, who have demonstrated an effect of salinity at the level of the Lap genotype in $M$. edulis, we would expect to find in these experiments that genotype specific mortalities would reduce the frequency of the $\operatorname{Lap}^{94}$ allele in the low salinity cultures. The only significant deviation from expected Lap allele frequencies in the single family occurred in the culture grown throughout at low salinity (M1D, table 1). At the spat stage in this culture there was a significant excess of the $L a p^{94}$ allele (expected frequency $0 \cdot 250$, observed frequency $0 \cdot 328$ ) but by the time the culture was sampled at the juvenile stage there was a significant deficiency of the $L a p^{94}$ allele (observed frequency 0.190). The difference between the allele frequencies in the two samples was significant at the $0 \cdot 1$ per cent level $\left(\chi_{2}^{2}=16 \cdot 942\right.$ $P<0.001)$. This temporal change in allele frequencies was principally due to a large change in the frequency of the genotype $\operatorname{Lap}^{94 / 98}$ while $L a p^{94 / 96}$ was present at approximately the expected frequency at the juvenile stage.

Mussels from the equivalent mass mating culture (MMD) also exhibited a lower frequency of $\operatorname{Lap}^{94}$ than expected at the juvenile stage and further mortalities during the periods of emersion produced a significant deficiency of $\operatorname{Lap}^{94}$ (table 2 ). In contrast to the single family culture, however, a low frequency of the $L a p^{94 / 96}$ genotype contributed strongly to the $L a p^{94}$ deficiency while the $\operatorname{Lap}^{94 / 98}$ genotype occurred at close to expected frequency.

Although culture MMD was not sampled at the spat stage, data are presented for culture MMC which was reared to the spat stage at low salinity. In this culture (table 2), the Lap $^{94}$ allele is significantly in excess at the spat stage with all three Lap ${ }^{94}$ genotypes occurring at higher than expected frequencies. This follows the pattern observed in the single family where low salinity during the larval stage tends to favour $\operatorname{Lap}^{94}$.

In the cultures which were reared initially at high salinity and later reduced to low salinity (MIB and $\mathrm{MMB}$ ) estimates of allele frequencies suggest a reduction of $L a p^{94}$ frequency between the spat and juvenile samples (MIB spat $0 \cdot 312$, juveniles $0 \cdot 225$; MMB spat $0 \cdot 135$, Juveniles $0 \cdot 108$ ) though in neither case was the change significant (MIB, $\chi^{2}=5.88 \quad P<0.10>0.05 ; \mathrm{MMB}, \chi^{2}=0.783 \quad P<$ $0 \cdot 70>0 \cdot 50$ ).

These results at the Lap locus, in general, provide supporting evidence for the conclusions reached by Hilbish and Koehn (1985a) that individual juvenile mussels carrying the $L a p^{94}$ allele, either as a homozygote or a heterozygote, are less fit than other genotypes and have a higher mortality in conditions of low salinity. However, our data also suggest that the fitness of individuals carrying the $L a p^{94}$ allele appears to be higher than 
other individuals at low salinities during the larval stage.

It is possible that the mechanism for cell volume regulation under hyperosmotic stress may be different in larvae compared to adults. Alternatively other mechanisms may operate during the larval stage which compensate for the higher protein catabolism postulated by Hilbish and Koehn $(1985 b)$ to occur in adult individuals carrying the Lap $^{94}$ allele. It is known that lipids provide the major energy reserves in larvae compared to glycogen in adults of certain bivalves (Gabbott, 1983) and it is therefore possible that the relative importance of other metabolic and catabolic pathways may differ considerably between the larval and the adult stage.

If, for whatever reason, certain Lap alleles are favoured differently at different times during the life cycle of the mussel then this could help to explain how a balanced Lap polymorphism is maintained, as long as heterozygotes have an overall fitness advantage. The particular situation of the $L a p^{94}$ allele frequency cline in Long Island Sound is thought to rely principally upon an annual influx of cohorts of larvae derived from an oceanic population of mussels with a high frequency of $\operatorname{Lap}^{94}$ (Koehn, 1985). However, if $\operatorname{Lap}^{94}$ is favoured at low salinity during the larval stage, it is possible to postulate some further recruitment from within the sound by cohorts of larvae derived from indigenous populations with low $L a p^{94}$ frequency.

Although we have so far made the assumption that the observed allele frequency changes at the Lap locus are related directly to salinity changes it is possible that selection is operating indirectly on some other locus or other loci closely linked to, or on the same chromosome as the Lap locus. The experimental protocol used does not enable us to distinguish between direct and indirect selection at the loci analysed and indeed, as will emerge later, indirect selection is perhaps more likely than direct selection at some of the other loci used in this study. Nevertheless Hilbish and Koehn (1985) and Koehn (1985) have provided strong evidence that the Lap locus in mussels is directly affected by salinity changes and it therefore seems appropriate to discuss our results in the light of this evidence.

A consistent picture emerges at the Pgm locus in the single family. In every culture, irrespective of salinity regime, there is a significant excess of the $P g m^{92}$ allele at the juvenile stage, but no significant variations in allele frequency at the spat stage. The allele $\mathrm{Pgm}^{92}$ occurs only with $\mathrm{Pgm}^{100}$ in the heterozygous form in the single family and clearly individuals homozygous for $\mathrm{Pgm}^{92}$ are fitter than the $P \mathrm{Pm}^{100 / 92}$ heterozygotes. In the mass mating cultures data are not available at the Pgm locus at the spat stage, but significant deviations from expected allele frequencies occur at the juvenile stage in most cultures. However, in these cases, it is the $\mathrm{Pgm}^{80}$ allele which is generally in excess, not $\mathrm{Pgm}^{92}$. In the mass mating, where the alleles $\mathrm{Pgm}^{92}$ and $\mathrm{Pgm}^{100}$ occur as homozygotes, as the heterozygote $\mathrm{Pgm}^{100 / 92}$, and also in combination with $\mathrm{Pgm}^{80}, \mathrm{Pgm}^{85}, \mathrm{Pgm}^{108}$ and $\mathrm{Pgm}^{116}$, the $\mathrm{Pgm}^{92 / 92}$ genotype does not appear to be consistently more fit than other genotypes.

There are three possible explanations for these observations. Firstly, the apparent significant excess of the $\mathrm{Pgm}^{92 / 92}$ genotypes in the single family may be due to mis-scoring heterozygotes as homozygotes. We think this is highly unlikely because the occasional presence and misinterpretation of satellite bands on gels scored for Pgm would lead to an excess of heterozygotes rather than homozygotes. Besides, the Pgm locus is normally clearly resolved and a very high degree of mis-scoring must be postulated to account for the large differences observed in all cultures.

Secondly, the relative fitness of the $\mathrm{Pgm}^{92 / 92}$ genotype may be different when in competition with only one other genotype rather than with many genotypes. However, the Pgm genotypes in the mass mating do not rank consistently for fitness irrespective of salinity regime as they do in the single family.

The third, and in our view, most likely explanation is that the Pgm locus is acting in the single family as a marker for another locus, or particular combination of loci, on the same chromosome which has a strong effect on fitness. In the mass mating the offspring are the products of many chromosomes and therefore particular Pgm genotypes are not as likely to act as markers for cis-linked loci unless they are located very close to the Pgm locus. In a previous comparison between two half-sib families of mussels (Beaumont et al., 1983) the genotype $P_{g m^{100 / 92}}$ occurred at expected frequencies in one family, but was found to be significantly deficient in the other. This adds support to the hypothesis that selection is acting at another locus on the same chromosome for which the Pgm locus acts only as a marker.

Neither genotype nor allele specific mortalities are associated with a particular salinity regime at the Gpi locus. Furthermore, at the juvenile stage in the single family, $G p i^{5}$ deficiency is generally an important component of the significant devi- 
ations from expected allele frequencies while juvenile offspring from the mass mating carrying the $G p i^{5}$ allele are generally in excess. This suggests that the Gpi locus may be similar to the Pgm locus in that it acts as a marker for another locus on the same chromosome. The Odh, Pgk and Dia loci also show significant deviations from expected allele frequencies in a number of cultures but in no case is there a consistent relationship between the frequency of particular alleles and the salinity at which the cultures were reared.

Some of the significant values for the $\chi^{2}$ goodness of fit tests comparing expected and observed allele or genotype frequencies will have arisen by chance alone. In total, 154 separate $\chi^{2}$ tests were performed and of these perhaps eight significant values at $P<0.05$, two at $P<0.01$ and one at $P<0.001$ might be expected to have arisen by chance. There were in fact $31 \chi^{2}$ tests which showed a significance at $P<0.05,25$ at $P<0.01$ and 26 at $P<0.001$ and the data therefore clearly show evidence of considerable overall selection.

Further confirmation that some form of nonrandom mortality of genotypes took place during the course of the experiments is provided by the significant overall heterogeneity of genotype frequencies across all cultures revaled at every locus. Interestingly, the results do not suggest that less heterogeneity was evident at the spat stage compared to the juvenile stage at all loci. At certain loci, for example, Pgm, significant non-random mortality in the single family was only evident after the spat stage and, because the same genotype was favoured in all cultures, there was no significant heterogeneity at either the spat or the juvenile stages. At other loci other situations were apparent; significant heterogeneity at both stages, significant heterogeneity at the spat stage but not at the juvenile stage, and vice versa. There was therefore no clear evidence that non-random mortality had occurred progressively with time indicating that selective forces had probably operated at different times during the experiment and that most loci were affected differently.

The complexity of the results and the fact that any selection was apparently unrelated to salinity at all loci, except possibly Lap, poses a problem. From an ecological viewpoint the salinity variations applied to the mussels reared in our experiments were very small and were therefore perhaps unlikely to have had a strong effect. Nevertheless, in a system where larvae and spat were reared at constant temperature under constant illumination and with similar volumes of algal food added, what could be the strong selective factors which pro- duced such significant variations in genotype frequencies between cultures? The only factor which we did not attempt to regulate in the laboratory cultures was density, beyond ensuring that each culture began with equal numbers of larvae. It was evident during final sampling that there were considerable differences in the density of mussels between cultures at the juvenile stage. However, regular examination of the condition of larval cultures up to metamorphosis revealed little overall larval mortality. It is nevertheless possible that mortalities at, or soon after metamorphosis may have produced significant density differences between cultures before sampling at the spat stage. We think that this is unlikely because samples of empty post-larval shells retrieved at the end of the experiments from several cultures indicated that most mortalities occurred after the spat sampling stage. Significant density-dependent genetic effects have recently been demonstrated in the relationship between multiple locus heterozygosity and growth rate in mussels (Gentili and Beaumont, 1988). Nevertheless, even if density dependent selection were operating in these experiments, it is still necessary to postulate some factor which caused the significant variation in mortality between cultures irrespective of salinity, and, for this, we can offer no satisfactory explanation.

Although these data have provided strong evidence for some form of selection affecting several loci in labratory reared cultures of mussels, the apparent complexity of the results and the problems of interpretation of the data mitigate against the use of laboratory cultures to address problems in ecological genetics. On the other hand, the evidence presented here of significant nonrandom mortality in the face of apparently weak selective forces, should warn us of the possible effects of the more extreme ecological variations which are likely to be found in the wild.

Acknowledgements This work was supported by a Natural Environment Research Council grant GR3/5286 to ARB. We thank two anonymous referees for valuable criticism of an earlier draft of this paper. We are grateful to Mr B. Roberts for the maintenance of cultures of microalgae.

\section{REFERENCES}

ADAMKEWICZ, L., TAUB, S. R. AND WALL, J. R. 1984. Genetics of the clam Mercenaria mercenaria. II. Size and genotype. Malacologia 25, 525-533.

AHMAD, M., SKIBINSKI, D. O. F. AND BEARDMORE, J. A. 1977. An estimate of the amount of genetic variation in the common mussel Mytilus edulis. Biochem. Genet. 15, 833846. 
BAYNE, B. L. 1965. Growth and the delay of metamorphosis of the larvae of Mytilus edulis (L.). Ophelia 2, 1-47.

BEAUMONT, A. R., BEVERIDGE, C. M. AND BUDD, M. D. 1983. Selection and heterozygosity within single families of the mussel, Mytilus edulis. (L.). Mar, Biol. Letts. 4, 151-161.

BEAUMONT, A. R. AND BEVERIDGE, C. M. 1983. Resolution of phosphoglucomutase isozymes in Mytilus edulis L. Mar. Biol. Letts. 4, 97-103.

BEAUMONT, A. R., DAY, T. R. AND GADE, G. 1980. Genetic variation at the octopine dehydrogenase locus in the adductor muscle of Cerastoderma edule (L.) and six other bivalve species. Mar. Biol. Letts. 1, 137-148.

DIEHL, W. J., GAFFNEY, P. M., MCDONALD, J. H. AND KOEHN, R. K. 1985. Relationship between weight-standardised oxygen consumption and multiple-locus heterozygosity in the marine mussel Mytilus edulis L. (Mollusca). In Gibbs, P. E., Proc. Eur. Mar. Biol. Symp., Cambridge University Press, pp. 529-534.

GABвоTT, P. A. 1983. Developmental and seasonal metabolic activities in marine molluscs. In Hochachka, P. W. (ed) The Mollusca. Vol. 2. Environmental Biochemistry and Physiology, Academic Press, pp. 165-217.

GAFFNEY, P. M. AND SCOTT, T. M. 1984. Genetic heterozygosity and production traits in natural and hatchery populations of bivalves. Aquaculture 42, 289-302.

GENTILI, M. R. AND BEAUMONT, A. R. 1988. Environmental stress, heterozygosity and growth rate in Mytilus edulis. $J$. Exp. Mar. Biol. Ecol, (In press).

HARRIS, H. AND HOPKINSON, D. A. 1976. Hand Book of Enzyme Electrophoresis in Human Genetics. North Holland Pub. Co., Amsterdam. 259pp.

HILBISH, T. J. AND KOEHN, R. K. $1985 a$. Dominance in physiological phenotypes and fitness at an enzyme locus. Science $229,52-45$.

Hilbish, T. J. AND KOEHN, R. K. 1985b. Genetic variation in nitrogen metabolism in Mytilus edulis: contribution of the Lap locus. In Gibbs, P. E. (ed.) Proc. 19th Eur. Mar. Biol. Symp., Cambridge University Press, pp. 497-504.

HVILSOM, M. M. AND THEISEN, B. F. 1984. Inheritance of allozyme variations through crossing experiments with the blue mussel, Mytilus edulis L. Hereditas, 101, 1-7.
KOEHN, R. K. 1983. Biochemical genetics and adaptation in molluscs. In Hochachka, P. W. (ed.) The Mollusca, Vol. 2. Environmental Biochemistry and Physiology, Academic Press, pp. 305-330.

KOEHN, R. K. 1985. Adaptive aspects of biochemical and physiological variability. In Gibbs, P. E. (ed.) Proc. 19th Eur. Mar. Biol. Symp., Cambridge University Press, pp. 425-441.

KOEHN, R. K. AND GAFFNEY, P. 1984. Genetic heterozygosity and growth rate in Mytilus edulis. Mar. Biol. 82 1-7.

KOEHN, R. K., Milkman, R. AND MitTon, J. B. 1976. Population genetics of marine pelecypods. IV. Selection, migration and genetic differentiation in the blue mussel Mytilus edulis. Evolution 30, 2-32.

LEVINTON, J. S. \& FUNDILLER, D. 1975. An ecological and physiological approach to the study of biochemical polymorphisms. In Barnes, H. (ed.) Proc. Ninth Europ. Mar. Biol. Symp., Aberdeen Univ. Press, Aberdeen, pp. 165-176.

MALLET, A. L., ZOUROS, E., GARTNER-KEPKAY, K. E., FREEMAN, K. R. AND DICKIE, L. M., 1985. Larval viability and heterozygote deficiency in populations of marine bivalves: evidence from pair matings of mussels. Mar. Biol. 87, 109-123.

MALLET, A. L. ZOUROS, E., GARTNER-KEPKAY, K. E. AND FREEMAN, K. R. 1986. Genetics of growth in blue mussels: family and enzyme-heterozygosity effects. Mar. Biol. 92, 475-482.

SKIBINSKI, D. O. F., BEARDMORE, J. A. AND CROSS, T. F. 1983. Aspects of the population genetics of Mytilus (Mytilidae; mollusca) in the British Isles. Biol. J. Linn. Soc. 19, 137-183.

SPRUNG, M. AND BAYNE, B. L. 1984. Some practical aspects of fertilising the eggs of the mussel Mytilus edulis L. J. Cons. Int. Explor. Mer., 41, 125-128.

TRACEY, M. L., BELLET, N. F. AND GRAVEM, C. D. 1975. Excess allozyme homozygosity and breeding population structure in the mussel Mytilus californianus. Mar. Biol. 32, 303-311.

TURNER, V. S. AND HOPKINSON, D. A. 1979. The use of meldola blue in isozyme stains after electrophoresis. F.E.B.S. Letters, 105, 376-378.

ZOUROS, E. AND FOLTZ, D. W. 1984. Possible explanations of heterozygote deficiency in bivalve molluscs. Malacologia, $25,583-591$. 